\title{
In vitro generation of CD4+CD25+ regulatory cells from murine naive $\mathrm{T}$ cells
}

\author{
Massimo C Fantini ${ }^{1}$, Sabine Dominitzki ${ }^{2}$, Angelo Rizzo ${ }^{1}$, Markus F Neurath ${ }^{2}$ \& Christoph Becker ${ }^{2}$ \\ ${ }^{1}$ Division of Gastroenterology, University of Rome Tor Vergata, Via Montpellier 1, 00133, Rome, Italy. ${ }^{2}$ Laboratory of Immunology, I. Medical Clinic, University of Mainz, \\ Obere Zahlbacher Strasse 63, 55131 Mainz, Germany. Correspondence should be addressed to C.B. (chbecker@uni-mainz.de).
}

Published online 12 July 2007; doi:10.1038/nprot.2007.258

CD4+CD25+ regulatory T cells (Tregs) are crucial for the maintenance of immunological tolerance. Recent data indicate that Tregs not only develop in the thymus during ontogeny but can also differentiate from naive T cells in the periphery. The following protocol describes a method by which Tregs are generated in vitro by stimulation of naive $T$ cells in the presence of transforming growth factor $\beta$ (Ti-Tregs). In vitro-induced regulatory $T$ cells express markers of conventional Treg such as CD25 and the genetic program committing transcription factor FoxP3. Functionally the in vitro-generated Ti-Tregs suppress T-cell activation and proliferation while in vivo these cells have been proven to control inflammation in different animal models, suggesting a potential use of these cells for immunotherapy. The protocol can be completed within 5 days.

\section{INTRODUCTION}

Many lines of evidence sustain that defects of Tregs play a pivotal role in the pathogenesis of many autoimmune diseases. Indeed these cells are thought to prevent the activation and expansion of autoreactive T-cell clones that have escaped the negative selection in the thymus ${ }^{1}$. Tregs are generated in the thymus after the first days of postnatal life and then migrate into peripheral organs where they can prevent the activation of autoreactive T-cell clones. Indeed thymectomy performed in mice at day 3 (3Tx) after birth, when regulatory $\mathrm{T}$ cells have not yet been released to the periphery, leads to the development of a multiorgan autoimmune disease that can be prevented by adoptive transfer of syngenic Tregs ${ }^{2}$. Recently, the winged helix transcription factor FoxP3 has been shown to play a key role in determining the regulatory phenotype of Tregs. Indeed, infection of naive $\mathrm{T}$ cells with a retroviral vector expressing FoxP3 was sufficient to induce a regulatory phenotype in these cells ${ }^{3}$. Moreover, deletion or loss of function of the FoxP3 encoding gene determines an autoimmune phenotype both in humans (the immune dysregulation, polyendocrinopathy, enteropathy, X-linked syndrome $)^{4}$ and mice (Scurfy) ${ }^{5}$. Functionally Tregs have been studied both in vitro and in vivo. In vitro, CD4+CD25+ Tregs have been shown to suppress the activation and expansion of naive cells, and it has been shown that this process does not require soluble factors but a direct contact of regulatory cells with target cells. The regulatory function of these cells has also been tested in different animal models of human disease. In models of colitis, Tregs showed the capacity to prevent and cure gut inflammation ${ }^{6}$. In addition, regulatory cells have been shown to efficiently prevent the development of experimental autoimmune encephalitis in mice, suggesting that these cells might have a potential role in the therapy of human disease ${ }^{7}$.

Despite data obtained so far on the function of Tregs, the study and the use of these cells for in vivo experiments has been limited by the exiguous number of Tregs normally present in the peripheral blood. Tregs represent only about $5-10 \%$ of the circulating CD4+ cells. Moreover, the in vitro expansion of these cells is hampered by their anergic phenotype. Indeed, naturally occurring Tregs poorly proliferate in response to TCR stimulation and need exogenous interleukin (IL)-2 to undergo expansion.

We and others have recently shown that cells endowed with regulatory capacity comparable to that of naturally occurring Tregs might be obtained upon activation of naive $\mathrm{T}$ cells in the presence of the cytokine TGF- $\beta^{8,9}$. This protocol describes the methods used in these papers in more detail. In this regard, experiments have been performed to fine-tune and troubleshoot the procedure, thus substantially improving the method initially described.

TGF- $\beta$ stimulation of CD4+CD25- cells obtained from a double-negative magnetic sorting for CD4 and CD25 has been shown to induce the expression of FoxP3 and many genes normally expressed by naturally occurring Tregs. These cells have been proven to prevent the activation and proliferation of naive cells in coculture experiments in vitro, while in vivo, Ti-Tregs have been shown to prevent the activation of the immune system in different models of human immune diseases ${ }^{8,10}$. In addition, TGF- $\beta$ has been implicated, together with IL- 6 , in the generation of a class of effector cells characterized by the expression of IL-17, called TH17 cells ${ }^{11,12}$. Therefore, TGF- $\beta$ emerges as a key molecule involved in the polarization of classes of effector cells exerting opposite roles depending on the cytokine milieu. In this paper, we illustrate the methods to generate fully competent Tregs starting from a population of naive $\mathrm{T}$ cells (CD4+CD25-). Tregs generated in this way can be used to study the generation and function of this crucial cell population and can further be used to investigate the potential use of Tregs for immunotherapy.

\section{MATERIALS}

REAGENTS

- BALB/c mice: 6- to 8-week-old BALB/c mice were obtained from the animal facility of the University of Mainz ! CAUTION All animal experiments must be performed in accordance with national and institutional regulations.
-Anti-mouse CD3 (145-2C11) and anti-mouse CD28 (37.15) antibody. They were affinity purified from hybridoma cell lines kindly provided by Professor E. Schmitt (Institute for Immunology, Johannes Gutenberg University, Mainz, Germany), using protein G-Sepharose (Pharmacia Biotech) 
-Anti-mouse FoxP3-APC, clone FJK-16s (eBioscience)

- FoxP3 intracellular immunofluorescence staining kit

(eBioscience)

- ACK cell lysis buffer (Cambrex)

- Recombinant TGF- $\beta 1$ (R\&D Systems)

- Recombinant IL-2 (R\&D Systems)

- Serum-free medium X-Vivo15, RPMI supplemented with 10\% (v/v)

FBS (BioWhittaker)

- CD4 cell isolation kit (Miltenyi Biotech)

- CD25 micro beads kit (Miltenyi Biotech)

- Celltrace CFSE (Invitrogen)

- Mitomycin C (Sigma-Aldrich)

- TRK lysis buffer, for real-time (RT-PCR) only

- Hi-Bind-RNA columns (peqGOLD Total RNA Kit, Peqlab), for RNA isolation for RT-PCR only

-M-MLV reverse-transcriptase (Invitrogen) for RT-PCR only
- iQ sybr green mix (Bio-Rad), for RT-PCR only EQUIPMENT

- LD columns and LS columns (Miltenyi Biotec)

- MidiMACS Separator (Miltenyi Biotec)

- MACS MultiStand (Miltenyi Biotec)

- FACSCalibur (Becton Dickinson)

- Scissors and forceps

- Tubes $50 \mathrm{ml}$

- Petri dish

- Microscope glass slides

- Pipettes

- Centrifuge

- Microscope for cell counting

- Burker or Neubauer cell count chambers

-24- and 96-well plates

- Real-time thermocycler, for RT-PCR only

\section{PROCEDURE}

\section{Splenocyte isolation}

1| Kill either male or female 6- to 8-week-old mice by cervical dislocation.

$\triangle$ CRITICAL STEP Perform all steps in the procedure under a sterile laminar flow hood.

$\triangle$ CRITICAL STEP Clean scissor and forceps properly with water and soap, and keep them in a beaker containing $70 \%$ ethanol.

2| Rinse the whole mouse thoroughly with $70 \%$ ethanol in order to realize a first line of disinfection.

3| With the mouse in supine position, open the abdomen by a first transversal cut avoiding opening of the peritoneal cavity. Completely expose the peritoneal sac by pulling the skin in opposite directions using your hands or forceps. At this point, the spleen should become visible on the left side of the mouse. With a second cut, open the peritoneal sac and collect the spleen with sterile forceps.

$\triangle$ CRITICAL STEP Avoid contact between the spleen and the mouse skin to preserve sterility.

4| Clear the spleen from residual debris and fat tissue and place it in a $50 \mathrm{ml}$ tube containing $30 \mathrm{ml}$ of $1 \times$ PBS. Wash the spleen twice with fresh PBS and subsequently transfer it into a $10 \mathrm{~cm}$ Petri dish containing $20 \mathrm{ml}$ of complete RMPI medium.

5| By using microscope glass slides previously sterilized with $70 \%$ ethanol and fire, cut spleens in two halves and squeeze them between the slides to extract the cellular matrix contained within the spleen capsule (Fig. 1). Discard the remaining fibrous tissue.

6| Collect the cell aggregates obtained from this step in a $50 \mathrm{ml}$ tube and pipette up and down several times by using a conventional pipettor to obtain a homogeneous cell suspension. Centrifuge the cell suspension at $400 \mathrm{~g}$ for 6 min at $4{ }^{\circ} \mathrm{C}$ in order to pellet the cells. Discard the supernatant.

\section{Lysis of erythrocytes}

7| Lyse red cells by resuspending the cell pellet in $5 \mathrm{ml} \mathrm{ACK}$ buffer for $90 \mathrm{~s}$.

8| Immediately afterwards, dilute the cell suspension with $45 \mathrm{ml}$ of $1 \times$ PBS to neutralize the ACK buffer. Spin cells in a centrifuge at $400 \mathrm{~g}$ for $10 \mathrm{~min}$ at $4{ }^{\circ} \mathrm{C}$ in order to pellet the cells and subsequently resuspend the pellet in $10 \mathrm{~mL}$ of $1 \times$ PBS. The cellular pellet should appear whitish, indicating successful lysis of red cells.

9| Count splenocytes by using a conventional Neubauer chamber to prepare them for the magnetic sorting steps. To do this, pipette $10 \mu$ of cell suspension between the chamber and the cover glass slide. Count the cells contained in the four corner quadrants. The number obtained, divided by 4 gives the number of cells per ml multiplied by $10^{4}$.
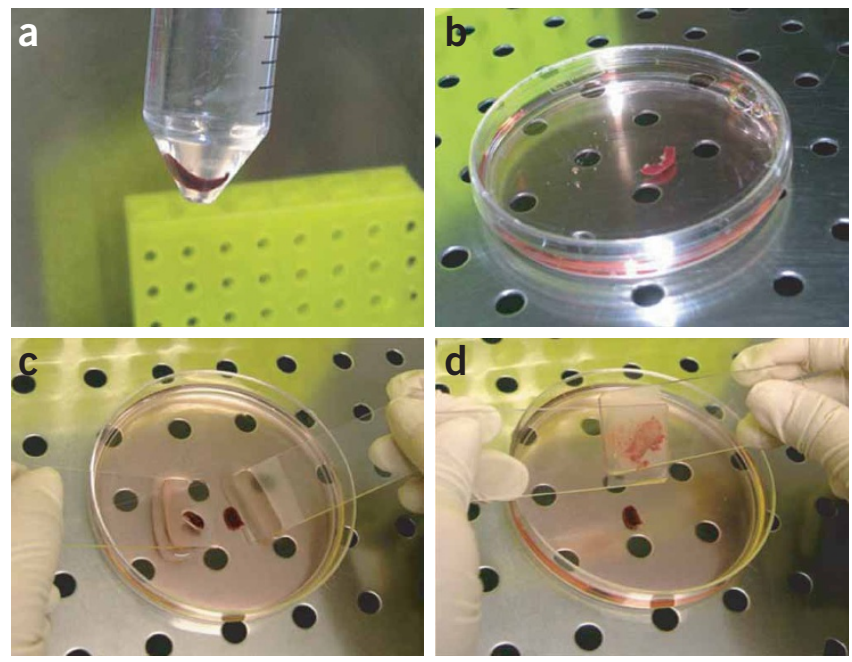

Figure 1 | Splenocytes isolation. Place the spleen in a Petri plate with $20 \mathrm{ml}$ of RPMI $10 \%$ FBS, cut in two pieces and squeeze each of them between two sterile glass slides to obtain a single cell suspension. (a) The isolated spleen is placed in a $50 \mathrm{ml}$ tube containing PBS. (b) The spleen is transferred into a $10 \mathrm{~cm}$ Petri dish containing $20 \mathrm{ml}$ of complete RMPI medium. (c) By using microscope glass slides previously sterilized with $70 \%$ ethanol and fire, the spleens are cut in two halves and (d) squeezed between the slides in order to extract the cellular matrix contained within the spleen capsule. 
Magnetic sorting of CD4+CD25 - cells: part 1

10| If using $10^{8}$ splenocytes as the starting cell number, use the Miltenyi CD4 cell isolation kit to label splenocytes from

Step 9 with $100 \mu \mathrm{l}$ of the biotinylated cocktail of antibodies specific for CD4-negative cells diluted in $400 \mu \mathrm{l}$ of RPMI $10 \%$ FBS for 15 min at $4{ }^{\circ}$.

11| Add $200 \mu$ l of the anti-biotin antibody conjugated with microbeads and $300 \mu \mathrm{l}$ of RPMI $10 \%$ FBS and incubate for 10 min at $4{ }^{\circ} \mathrm{C}$.

12| After labeling, wash cells once with RPMI $10 \%$ FBS and resuspend in $6 \mathrm{ml}$ of RPMI containing 10\% FBS.

13| Separate untouched CD4+ cells by loading $2 \mathrm{ml}$ of the labeled cells on to an LD separation column (Miltenyi). CD4-negative cells will remain in the column owing to the magnetic beads attached to their surface. CD4-positive cells, in contrast, will flow through the column and can be collected in a tube (Fig. 2a). Repeat twice, loading an additional $2 \mathrm{ml}$ of cells each time. Count CD4+ cells as indicated in Step 9.

\section{Magnetic sorting of CD4+CD25 - cells: part 2}

14| In the second part, label the untouched CD4+ cells (cells that flowed through the column in Step 13) with an anti-

CD25PE antibody (Miltenyi; allow $10 \mu$ of antibody for every $10^{6}$ CD4+ cells diluted in $100 \mu$ l of RPMI $10 \%$ FBS). Incubate the cells for 15 min at $4^{\circ} \mathrm{C}$.

15| Wash cells once with $10 \mathrm{ml}$ of RPMI $10 \%$ FBS.

16 Incubate cells with anti-phycoerythrin (PE) antibody conjugated with microbeads (Miltenyi; allow $10 \mu \mathrm{l}$ of antibody for every $10^{6} \mathrm{CD} 4+$ cells diluted in $90 \mu \mathrm{l}$ of RPMI $10 \% \mathrm{FBS}$ ). Incubate the cells for 15 min at $4^{\circ} \mathrm{C}$.

17| Resuspend cells in $6 \mathrm{ml}$ of complete medium. Separate CD4+CD25 - cells from CD4+CD25+ cells by loading an LS positive selection column (Miltenyi) $1 \mathrm{ml}$ at a time. Wait for cells to go through completely before loading the second $\mathrm{ml}$. Collect the flow through containing the untouched CD4+CD25- cells (Fig. 2a).

$\triangle$ CRITICAL STEP Although the use of an LS column in this depletion step is not suggested by the manufacturers, as the number of CD25+ cells to be sorted out does not normally exceed 5-10\% of CD4+ population, the use of an LS in place of an LD depletion column still allows an optimal depletion of CD25+ cells while increasing the final yield of CD4+CD25 - cells, which is generally lower using an LD depletion column. A typical yield is about $10^{6}$ CD4+CD25- cells per $10^{8}$ splenocytes.

\section{Flow cytometric purity analysis}

18 Stain a fraction of cells with anti-CD4 and anti-CD25 antibodies conjugated with fluorescein isothiocyanate and PE. For this we recommend $2.5 \times 10^{5} \mathrm{CD} 4+\mathrm{CD} 25$ - cells are sampled, washed once with PBS and resuspended in $100 \mu \mathrm{l}$ of PBS $0.2 \%$ BSA. Add $1 \mu \mathrm{l}$ of each antibody to the sample (1:100 dilution) and incubate the sample for 10 min at $4{ }^{\circ} \mathrm{C}$.

19| At the end of the incubation, wash the cells and analyze by flow cytometry to quality control your isolation procedure. In general, the purity of CD4+CD25- cells is more than $96 \%$ (not shown) according to the manufacturer's data sheet. Note that when sorted cells are analyzed for FoxP3 expression, only 2-3\% of the CD4+CD25- cells are FoxP3+ (Fig. $2 \mathbf{b}$ ), as previously described ${ }^{13}$. However, the possibility that this small number of FoxP3-expressing cells could be responsible for the TGF- $\beta$ mediated induction of FoxP3+ has been ruled out by experiments performed in TCR transgenic mice on a RAG1 knockout background, which do not have FoxP3-expressing cells, thus demonstrating that TGF- $\beta$ directly acts on naive cells ${ }^{14}$.

\section{Induction of Ti-Tregs}

20| Coat a 24-well plate with $10 \mu \mathrm{g} \mathrm{ml}^{-1}$ of anti-CD3 antibody in PBS at $37{ }^{\circ} \mathrm{C}$ for $2 \mathrm{~h}$ and then wash the plate once with PBS before cell plating.

21 Plate $1 \mathrm{ml}$ of $2 \times 10^{6} \mathrm{ml}^{-1} \mathrm{CD} 4+C D 25$ - cells resuspended in X-Vivo15 serum-free medium in the anti-CD3-precoated wells in the absence of antibiotics. Immediately add anti-CD28 antibody $\left(2 \mu \mathrm{g} \mathrm{ml}^{-1}\right)$ and TGF- $\beta 1\left(5 \mathrm{ng} \mathrm{ml}^{-1}\right)$ and incubate the cells for 5 days (Fig. 3 ).

? TROUBLESHOOTING 
Quantitative and qualitative evaluation of Ti-Tregs induction 22| After 5 days of incubation, test an aliquot of Treg culture for Foxp3 induction: we generally evaluate Foxp3 induction quantitatively either at the RNA or protein level by real-time PCR (option A) or flow cytometry (option B), respectively. We also recommend that you perform qualitative evaluation, and the suppressive capacity of Ti-Tregs can be evaluated in a coculture suppression assay (option C).

\section{(A) RT-PCR to detect FoxP3}

(i) Lyse $5 \times 10^{5}$ Ti-Tregs in TRK lysis buffer and isolate RNA; we use Hi-Bind-RNA columns according to the manufacturer's protocol.

(ii) Perform reverse transcription; we use M-MLV reverse- transcriptase (Invitrogen).

(iii) Perform real-time PCR. We generally use the following specific primers: mFoxP3 exon 6 sense $5^{\prime}$-GGTACACCCAGGAAAGAC AG-3' and mFoxP3 exon 9 antisense 5'-ATCCAGGAGATGATCTGCTTG-3'; plus iQ sybr green mix (Bio-Rad). We use an iQ5 real-time thermocycler to perform real-time PCR.

\section{(B) Flow cytometry to detect FoxP3}

(i) To determine the amount of FoxP3+ (regulatory) cells in the population, stain $2.5 \times 10^{5}$ cells with a PE-conjugated antiCD4 as previously described (Steps 18 and 19).

(ii) Fix, permeabilize and stain the cells with a specific APC-conjugated anti-FoxP3 antibody according to the instructions provided in the mouse FoxP3 staining kit (eBioscience).

(iii) Analyze stained cells by flow cytometry (Fig. 4).

\section{? TROUBLESHOOTING}

\section{(C) Qualitative evaluation of induced Tregs}

(i) Use freshly isolated CD4+CD25 - cells obtained by magnetic sorting, as described in Steps 10-17.

(ii) Label freshly isolated CD4+CD25 - cells with CFSE according to standard protocols: briefly, resuspend cells in PBS at a concentration of $10^{6}$ cells ml-1 and stain with $0.2 \mu \mathrm{M}$ CFSE for $1 \mathrm{~h}$ at $37^{\circ} \mathrm{C}$ wrapped with an aluminum foil to protect from light.

(iii) Wash cells once in PBS, resuspend at the same concentration in RPMI $10 \%$ FBS and incubate for a further hour at $37{ }^{\circ} \mathrm{C}$ to remove the unbound CFSE.

(iv) Plate these cells as responder cells at a concentration of $10^{5}$ cells well $^{-1}$ in a 96 -well round bottom plate together with MHC-matched APC cells $\left(2 \times 10^{4}\right.$ cells well $\left.{ }^{-1}\right)$, which have already been either treated with mitomycin $\mathrm{C}\left(60 \mu \mathrm{g} \mathrm{ml}^{-1}\right)$ for 30 min or irradiated $(4,000 \mathrm{Rad})\left(2 \times 10^{4}\right.$ cells well $\left.^{-1}\right)$.

(v) Add anti-CD3 to each well at the concentration of $1 \mu \mathrm{g} \mathrm{ml}^{-1}$. Add Ti-Tregs to the wells at different ratios as indicated in Figure 5a. Freshly isolated CD4+CD25+ Tregs and CD4+CD25 - cells activated in the absence of TGF- $\beta$ for 5 days can be used as positive and negative controls, respectively.
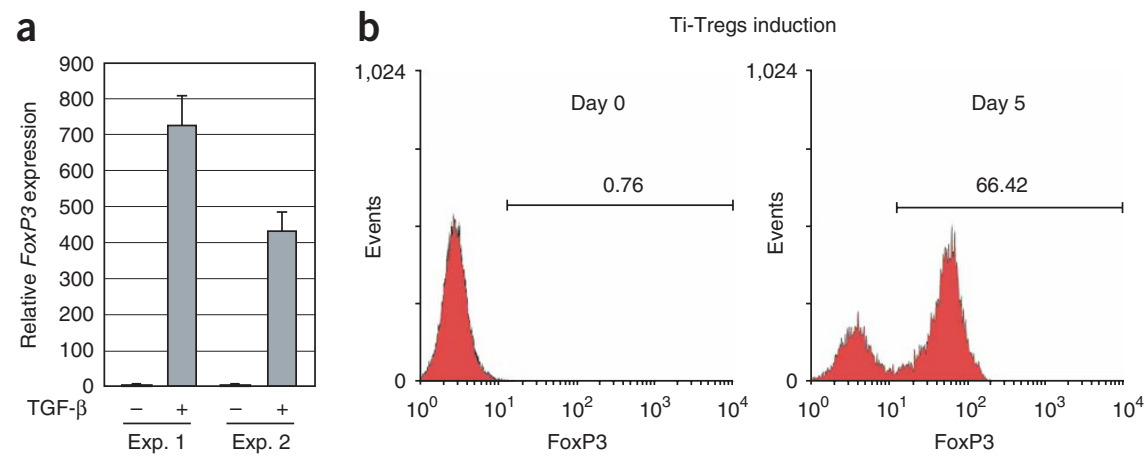

Figure 4 | Example of results obtained for Ti-Treg induction control. In (a), the expression of FoxP3 mRNA was evaluated in CD4+CD25 - cell culture activated for 5 days with plate-bound anti-CD3 and soluble anti-CD28 (2 $\left.\mu \mathrm{g} \mathrm{ml}^{-1}\right)$ in the presence or absence of TGF- $\beta$. Two representative Ti-Treg inductions are reported, which show an upregulation of FoxP3 in the range of 400- to 700-fold compared with cells activated in the absence of TGF- $\beta$ as quantified by real-time PCR. The same cells were evaluated by flow cytometry to quantify the number of cells expressing the transcription factor FoxP3. For this, an aliquot of day 5 Ti-Tregs was permeabilized and stained with an anti-mouse FoxP3-specific antibody. In (b), a representative flow cytometric analysis of day 5 Ti-Treg culture is shown. (vi) Analyze cells after 4 days by flow cytometry. As shown in Figure 5a, Ti-Treg significantly suppresses the proliferation of $\mathrm{CD} 4+\mathrm{CD} 25-$ responder cells at various concentrations indicated by the reduced CFSE dilution in these cultures. Quantification of cells undergoing divisions can be evaluated by determining the proliferation index obtained with the proliferation module of the ModFit LT Macintosh software

(Fig. 5b). ? TROUBLESHOOTING

\section{TIMING}

Steps 1-9, CD4+CD25- cell preparation: $4 \mathrm{~h}$

Steps 10-17, CD4/CD25 staining: $1 \mathrm{~h}$ 
PROTOCOL
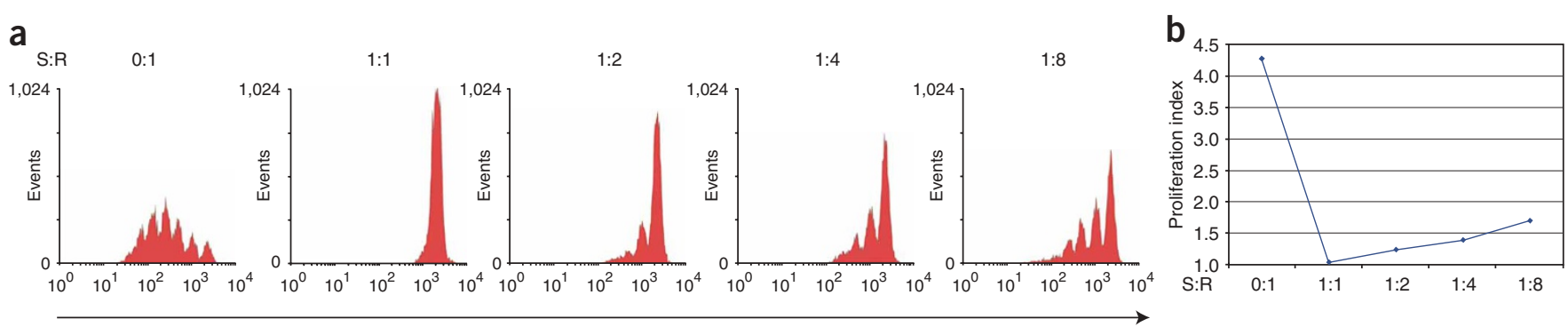

CFSE

Figure 5 | Example of Ti-Tregs functional quality control. To evaluate the suppressive capacity of Ti-Treg cultures, a proliferation assay was set. Briefly, freshly isolated CD4+CD25 - cells were used as responder cells upon CFSE staining. Responder cells were activated in the presence of soluble anti-CD3 $\left(1 \mu \mathrm{g} \mathrm{ml} \mathrm{m}^{-1}\right)$ and mytomicin-treated APC cells alone or in the presence of Ti-Tregs (suppressors) at different ratios as indicated. CFSE progressive dilution was used as readout of responder cells proliferation. As shown in (a), at all the responder:suppressor ratios, Ti-Tregs were able to suppress proliferation of responder cells. CFSE populations were also analyzed by determining the proliferation index obtained with the proliferation module of ModFit LT Macintosh software (b).

Step 21, Ti-Treg culture set up: $1 \mathrm{~h}$

Step 21, Ti-Treg induction: 5 days

Step 22A, RNA isolation: $2 \mathrm{~h}$; reverse transcription: $2 \mathrm{~h}$; real-time PCR: $3 \mathrm{~h}$

Step 22B, CD4/FoxP3 staining: $2 \mathrm{~h}$

Step 22C, CD4+CD25- isolation: 5 h; CFSE staining, $1 \mathrm{~h}$ and $30 \mathrm{~min}$; coculture set up: $1 \mathrm{~h}$; proliferation assay: 4 days

\section{? TROUBLESHOOTING}

\section{Step 21}

Cell death: High cell death in day $5 \mathrm{Ti}$-Treg culture might be observed upon propidium iodide staining. The use of serum-free medium in the absence of antibiotics increases the general cell viability. Alternatively, dead cells can be eliminated by using the Dead cell removal kit (Miltenyi Biotech), according to the manufacturer's protocol, at the end of the induction phase.

\section{Step 22B}

Low FoxP3 induction: Certain variability in the number of FoxP3-expressing cells obtained at the end of the induction phase might be observed in different animal strains (BALB/C versus $C B 57 B / 6$ ). This is, in our experience, mainly due to the different capacity of CD4+CD25 - cells to propagate in vitro for 5 days. To avoid a low yield of Ti-Tregs, we recommend adding $20 \mathrm{ng} \mathrm{ml}^{-1}$ of murine IL-2 to the cell cultures. This might improve the yield and viability of Ti-Tregs at the end of the induction phase (refs. 14,15; Fantini et al., unpublished observation).

\section{Step $22 \mathrm{C}$}

Contaminating FoxP3-negative cells: To enrich the population of Foxp3-expressing Ti-Tregs, an additional cell sorting for CD25 after resting for $24 \mathrm{~h}$ at the end of the induction culture may help eliminate a fraction of non-regulatory cells.

\section{ANTICIPATED RESULTS}

This protocol illustrates the procedure to generate CD4+CD25+FoxP3+ regulatory cells from a starting population of naive $T$ cells that are very similar to, if not indistinguishable from, naturally occurring Tregs developing in the thymus. The possibility to have an adequate number of cells available for experiments will help characterize the cellular mechanisms responsible for the suppressive activity of Tregs on target cells.

The peripheral generation of regulatory cells is sustained by several lines of evidence and TGF- $\beta$ has been implicated in this process. This protocol might represent an in vitro model of the process that physiologically leads to the peripheral generation of Tregs in vivo. At RNA level, the induction of FoxP3 in Ti-Tregs when compared to CD4+CD25 - cells activated in the absence of TGF- $\beta$ usually varies in a range of 400- to 700-fold while the number of Foxp3+ cells after 5 days of culture usually is around $60 \%$ (Fig. 4). Overall, the number of FoxP3+ cells recovered after 5 days is one- to twofold greater than the number of CD4+CD25-FoxP3 - cells originally plated. Functionally, Ti-Tregs suppress proliferation of freshly isolated CD4+CD25 - in a dose-dependent manner, similar to the suppression observed using naturally occurring CD4+CD25+ Tregs. Therefore, the study of the molecular events accompanying the induction of Ti-Tregs in vitro might shed light on new intracellular pathways involved in naive T-cell differentiation. The protocol described here can be easily modified to study the induction and function of antigen-specific regulatory T cells both in vitro and in vivo using T-cell receptor transgenic mice specific for a given antigen. Naturally occurring Tregs physiologically represent $5-10 \%$ of circulating CD4+ cells. Owing to the low number of naturally occurring Tregs obtained by sorting peripheral blood cells and because of the scarce expansion obtained in vitro, the availability 
PROTOCOL

of an adequate number of Tregs for in vivo experiments might often represent a limitation. The in vitro generation of Ti-Tregs has already demonstrated that these cells are effective in preventing disease in different animal models. The extension of these experiments to a curative setting will pave the way to a future use of these cells in those human diseases caused by an uncontrolled activation of the immune system. Indeed the capacity to induce regulatory cells in an antigen-specific manner and to transfer them in atopic patients might represent an important advance in the immunotherapy of allergic diseases.

COMPETING INTERESTS STATEMENT The authors declare no competing financial interests.

Published online at http://www.natureprotocols.com

Reprints and permissions information is available online at http://npg.nature.com/ reprintsandpermissions

1. Sakaguchi, S. et al. Foxp3+ CD25+ CD4+ natural regulatory T cells in dominant self-tolerance and autoimmune disease. Immunol. Rev. 212, 8-27 (2006).

2. Asano, M., Toda, M., Sakaguchi, N. \& Sakaguchi, S. Autoimmune disease as a consequence of developmental abnormality of a T cell subpopulation. J. Exp. Med. 184, 387-396 (1996).

3. Hori, S., Nomura, T. \& Sakaguchi, S. Control of regulatory T cell development by the transcription factor Foxp3. Science 299, 1057-1061 (2003).

4. Bennett, C.L. et al. The immune dysregulation, polyendocrinopathy, enteropathy, X-linked syndrome (IPEX) is caused by mutations of FOXP3. Nat. Genet. 27, 20-21 (2001).

5. Godfrey, V.L., Wilkinson, J.E. \& Russell, L.B. X-linked lymphoreticular disease in the scurfy (sf) mutant mouse. Am. J. Pathol. 138, 1379-1387 (1991).

6. Mottet, C., Uhlig, H.H. \& Powrie, F. Cutting edge: cure of colitis by CD4+CD25+ regulatory T cells. J. Immunol. 170, 3939-3943 (2003).

7. Kohm, A.P., Carpentier, P.A., Anger, H.A. \& Miller, S.D. Cutting edge: CD4+CD25+ regulatory $T$ cells suppress antigen-specific autoreactive immune responses and central nervous system inflammation during active experimental autoimmune encephalomyelitis. J. Immunol. 169, 4712-4716 (2002).

8. Chen, W. et al. Conversion of peripheral CD4+CD25 - naive T cells to CD4+CD25+ regulatory T cells by TGF-beta induction of transcription factor Foxp3. J. Exp. Med. 198, 1875-1886 (2003).

9. Fantini, M.C. et al. Cutting edge: TGF-beta induces a regulatory phenotype in CD4+CD25 - T cells through Foxp3 induction and down-regulation of Smad7. J. Immunol. 172, 5149-5153 (2004).

10. Fantini, M.C. et al. Transforming growth factor beta induced Foxp3+ regulatory T cells suppress Th1-mediated experimental colitis. Gut 55, 604-606 (2006).

11. Bettelli, E. et al. Reciprocal developmental pathways for the generation of pathogenic effector TH17 and regulatory T cells. Nature 441, 235-238 (2006).

12. Mangan, P.R. et al. Transforming growth factor-beta induces development of the $\mathrm{T}(\mathrm{H}) 17$ lineage. Nature 441, 231-234 (2006).

13. Zelenay, S. et al. Foxp3+ CD25- CD4 T cells constitute a reservoir of committed regulatory cells that regain $\mathrm{CD} 25$ expression upon homeostatic expansion. Proc. Natl. Acad. Sci. USA 102, 4091-4096 (2005).

14. Davidson, T.S., DiPaolo, R.J., Andersson, J. \& Shevach, E.M. Cutting Edge: IL-2 is essential for TGF-beta-mediated induction of Foxp3+ T regulatory cells. J. Immunol. 178, 4022-4026 (2007).

15. Zheng, S.G., Wang, J., Wang, P., Gray, J.D. \& Horwitz, D.A. IL-2 is essential for TGF-beta to convert naive CD4+CD25 - cells to CD25+Foxp3+ regulatory T cells and for expansion of these cells. J. Immunol. 178, 2018-2027 (2007). 